\title{
Prevalencia y ubicación de dientes supernumerarios
}

\author{
Location and Prevalence of Supernumerary Teeth
}

\section{Prevalência e localização de dentes supranumerários}

\author{
Marjory E. Vaca-Zapata' \\ marjoryvacazapata@gmail.com \\ Kleber A. Vallejo-Rosero" \\ avallejo@uce.edu.ec
}

Recibido: 12 de noviembre de 2016 * Corregido: 19 de diciembre de 2016* Aceptado: 4 de enero de 2017

'Odontóloga, Facultad de Odontología, Universidad Central del Ecuador, Quito, Ecuador.

IIDocente, Facultad de Odontología, Universidad Central del Ecuador, Quito, Ecuador. 


\section{Resumen.}

Objetivo: Conocer la prevalencia y ubicación de dientes supernumerarios en pacientes atendidos en el quirófano de la Facultad de Odontología de la Universidad Central Del Ecuador en el período 2010-2013. Materiales y metodos: El tipo de estudio de esta investigación es epidemiológico retrospectivo, no experimental de tipo transversal, la población estudiada correspondió a 831 historias Clínicas de pacientes que fueron atendidos en el Quirófano de la FOUCE, para la recolección de datos fue la observación directa y determinar si el paciente era considerado dentro del estudio, se analizaron la historia clínica y la radiografía, los resultados fueron almacenados y tabulados en el programa Microsoft Excel y sometidos a pruebas estadísticas descriptivas con el paquete de estadístico SPSS versión 10. Resultados: Se encontraron 34 pacientes con dientes supernumerarios, equivalentes al 4\%, 10 en mujeres $(29,4 \%)$ y 24 en hombres $(71 \%)$; la segunda década de vida comprendida entre los 11 y 20 años presentó 41,2\%. Se registró 20 dientes supernumerarios en el maxilar (57,14\%) y 15 en mandíbula (42,85\%). En la zona incisiva maxilar se reportó $47,1 \%$ de dientes supernumerarios, en la zona premolar 5,9\% y en la zona molar 5,9\%; en la región mandibular incisiva se registró el 2,9\%, en la zona canina el 2,9\%, en la zona premolar el 23,5\% y en la zona molar el 14,7\%. Conclusiones: Se estableció que los dientes supernumerarios se ubicaron con mayor frecuencia en maxilar que en mandíbula.

Palabras clave: Prevalencia; dientes supernumerarios; maxilar; mandíbula. 


\section{Abstract.}

Objective: Know the prevalence and location of supernumerary teeth in patients treated in the Universidad central del Ecuador Dental School operating room in 2010-2013. Materials and Methods: The type of study of this research is retrospective, non-experimental cross-sectional epidemiologic, the study population was 831 Clinical charts of patients who were treated in the Operating Room of the FOUCE. Data collection was performed by direct observation; and to determine if the patient was considered within the study, the clinical chart and the X-ray were analyzed. Results were stored and tabulated in Microsoft Excel and subjected to the SPSS version 10 statistical tests. Results: 34 patients were found with supernumerary teeth (4\%), 10 were women (29.4\%) and 24 were men (71\%); patients aged 11 to 20 represented $41.2 \%$. Twenty supernumerary teeth were present in the maxilla $(57.14 \%)$ and 15 in the mandible $(42.85 \%)$. In the maxillary incisor zone, $47.1 \%$ of supernumerary teeth were reported, in the premolar area $5.9 \%$ and in the molar zone 5.9\%; In the incisive mandibular area $2.9 \%$ were recorded, $2.9 \%$ in the canine zone, $23.5 \%$ in the premolar area and $14.7 \%$ in the molar zone. Conclusions: It was established that supernumerary teeth were located more frequently in the maxillary than in the mandible.

Keywords: Prevalence; Supernumerary teeth; maxillary; jaw. 


\section{Resumo.}

Objetivo: Conhecer a prevalência e localização dos dentes supranumerários em pacientes tratados no quirófano da Faculdade de Odontologia da Universidade Central do Equador no período 20102013. Materiais e Métodos: O tipo de estudo foi epidemiológico, retrospectivo, não experimental de tipo transversal, a população estudada foram 831 historias clinicas de pacientes tratados no quirófano da FOUCE, o método de recolecção de dados foi a observação direta para determinar se o paciente era considerado no estudo, analisaram-se a historia clinica e a radiografia, os resultados foram armazenados e catalogados no software Microsoft Excel e submetidos a testes estatísticos descritivos com o pacote estatístico SPSS versão 10. Resultados: Encontraram-se 34 pacientes com dentes supranumerários, equivalente a 4\%, 10 foram mulheres $(29,4 \%)$ e 24 homens (71\%); na segunda década de vida, entre os 11 e 20 anos, se apresentaram 41,2\% dos casos. Registraram-se 20 dentes supranumerários no maxilar $(57,14 \%)$ e 15 na mandíbula $(42,85 \%)$. Na zona incisiva maxilar reportaram-se $47,1 \%$ dos dentes supranumerários, na zona pré-molar 5,9\% e na zona molar 5,9\%; na região mandibular incisiva registraram-se 2,9\%, na zona canina 2,9\%, na zona pré-molar 23,5\% e na zona molar 14,7\%. Conclusões: Determinou-se que os dentes supranumerários se localizaram com maior frequência no maxilar do que na mandíbula.

Palavras chave: Prevalência, dentes supranumerários, maxilar, mandíbula 


\section{Introducción.}

La etiología de los dientes supernumerarios es considerada aún inexacta, aunque para muchos autores está relacionada con la división involuntaria del folículo del diente o bien sea por una hiperactividad de las células epiteliales embrionarias que componen a la lámina dentaria. (1)

Alvez N; et al, 2011, (2) menciona en su artículo que los dientes supernumerarios pueden ocasionar problemas en la dentición definitiva, tales como, el retraso de la erupción dentaria y mal posiciones dentarias, ocasionando malas oclusiones, reabsorción radicular de piezas vecinas y aparición de quistes. Además la hiperodoncia puede generarotras patologías que se presentan en síndromes como la disostosis cleidocraneal y en el síndrome de Gardner. (3)

Los dientes supernumerarios pueden presentarse en cualquier arcada dentaria, así los presentes en las zonas premaxilares llamados mesiodens permanecen retenidos en el tejido óseo en un $75 \%$, y erupcionanapenas el $25 \%$, mientras que en la dentición temprana se ha visto un incremento en la erupción de dicha anomalía con un 73\%. La utilización de métodos radiográficos tales como periapicales, panorámicas, oclusales estrictas superior o inferior, además de más métodos radiológicos nos ayudarán a identificarlos en caso de encontrarse retenidos. (4)

La ayuda fundamental, que aportan las radiografías panorámicas, pone en evidencia la necesidad de cada profesional de solicitar a cada uno de los pacientes que serán atendidos odontológicamente, una de ellas, para de esta manera brindarles un veraz diagnóstico y un óptimo tratamiento para su salud oral.

Este trabajo investigativo busca encontrar la frecuencia poblacional de dientes supernumerarios en pacientes atendidos en el Quirófano de la Facultad de Odontología de la 
Universidad Central del Ecuador, período 2010-2013; examinar los determinantes planteados tales como edad, género y la zona tanto maxilar como mandibular de mayor predilección, mediante el análisis de Historias Clínicas llenadas correctamente y la observación de las radiografías panorámicas.

\section{Materiales y métodos.}

El tipo de estudio de esta investigación es epidemiológico retrospectivo, no experimental de tipo transversal debido a que es un estudio en el cual se registró el número de pacientes que presentaron esta anomalía dentaria y acudieron al Quirófano de la F.O. de la U.C.E. durante el 2010 al 2013; observacional, ya que revisaremos las historias clínicas de dichos pacientes con sus respectivas radiografías panorámicas; descriptivo porque después de la recolección de los datos daremos una descripción de los mismos con el objeto de exponer nuestra población con esta anomalía dentaria; analítico ya que al final del estudio los resultados serán analizados con respecto a estudios similares y cuantitativo en cuanto a prevalencia ya que nuestro estudio pretende examinar exactamente la parte numérica de los datos obtenidos.

Para llevar a cabo el presente trabajo investigativo se dispone de los permisos correspondientes de las autoridades en función durante el desarrollo del trabajo para poder ingresar al quirófano de la FOUCE y tener acceso a las historias clínicas de pacientes atendidos en dicha institución, las mismas que me han proporcionado las radiografías panorámicas necesarias para este trabajo.

Las historias clínicas archivadas desde el 2010 hasta el 2013 y que cumplían con los criterios de inclusión fueron elegidas para la participación de este estudio. 
Con la ayuda de un Estadístico se realizó el análisis de los datos recolectados anteriormente, los mismos que previamente se encontraban ingresados de forma ordenada al programa de Microsoft Excel 2007. Para que el estadístico con programas como el SPSS 10 nos colabore con los procesos de tabulación porcentual con respecto a nuestras variables establecidas, los mismos que servirán para la realizaron de las tablas y gráficos, y gracias a esto lograr una mejor representación de los resultados.

\section{Resultados.}

El presente estudio tuvo una respuesta de 797 pacientes atendidos correspondiente al $96 \%$ NO presentaron dientes supernumerarios y 34 pacientes si los presentaron. (Tabla $\left.\boldsymbol{N}^{\circ} \mathbf{1}\right)$

PREVALENCIA

\begin{tabular}{ccccc}
\hline & FRECUENCIA & PORCENTAJE & PÓ RC ENTAJE \\
\hline NO & 797 & 95,9 & 95,9 & $\begin{array}{c}\text { POLO } \\
\text { ACUMULADO }\end{array}$ \\
SI & 34 & 4,1 & 4,1 & 95,9 \\
TOTA & 831 & 100,0 & 100,0 & 41,1 \\
& & &
\end{tabular}

Tabla $N^{\circ}$ 1.- Prevalencia de dientes Supernumerarios

\begin{tabular}{ccccc}
\multicolumn{5}{c}{ GENERO } \\
\hline & FRECUENCIA & PO RCENTAJE & $\begin{array}{c}\text { PORCENTAJE } \\
\text { VÁLIDO }\end{array}$ & $\begin{array}{c}\text { PO RC ENTAJE } \\
\text { ACUMULADO }\end{array}$ \\
\hline MASCULINO & 24 & 70,6 & 70,6 & 70,6 \\
FEMENINO & 10 & 29,4 & 29,4 & 29,4 \\
TOTAL & 34 & 100,0 & 100,0 & 100,0
\end{tabular}

Tabla $N^{\circ}$ 2.- Prevalencia de dientes supernumerarios de acuerdo al género 
EDAD EN AÑOS

\begin{tabular}{ccccc}
\hline & FRECUENCIA PORCENTAJE & $\begin{array}{c}\text { PORCENTAJE } \\
\text { VÁLIDO }\end{array}$ & $\begin{array}{c}\text { PORCENTAJE } \\
\text { ACUMULADO }\end{array}$ \\
\hline 1 a 10 & 6 & 17,6 & 17,6 & 17,6 \\
11 a 20 & 14 & 41,2 & 41,2 & 58,8 \\
21 a 30 & 12 & 35,3 & 35,3 & 94,1 \\
31 a 40 & 2 & 5,9 & 5,9 & 5,9 \\
TOTAL & 34 & 100,0 & 100,0 & 100,0
\end{tabular}

Tabla $N^{\circ}$ 3.- Prevalencia de dientes supernumerarios de acuerdo a grupos etarios

\begin{tabular}{ccccc}
\multicolumn{5}{c}{ MAXILAR } \\
\hline & FRECUENCIA & PO RCENTAJE & $\begin{array}{c}\text { PORCENTAJE } \\
\text { VÁLIDO }\end{array}$ & $\begin{array}{c}\text { PORCENTAJE } \\
\text { ACUMULADO }\end{array}$ \\
\hline No & 14 & 41,2 & 41,2 & 41,2 \\
Incisiva & 16 & 47,1 & 47,1 & 88,2 \\
Premolar & 2 & 5,9 & 5,9 & 94,1 \\
Molar & 2 & 5,9 & 5,9 & 5,9 \\
TOTAL & 34 & 100,0 & 100,0 & 100,0
\end{tabular}

Tabla $N^{\circ}$ 4.- Ubicación de dientes supernumerarios en maxilar

MANDIBULA

\begin{tabular}{ccccc}
\hline & \multicolumn{2}{c}{ FRECUENCIA PORCENTAJE } & $\begin{array}{c}\text { PORCENTAJE } \\
\text { VÁLIDO }\end{array}$ & $\begin{array}{c}\text { PORCENTAJE } \\
\text { ACUMULADO }\end{array}$ \\
\hline No & 19 & 55,9 & 55,9 & 55,9 \\
Incisiva & 1 & 2,9 & 2,9 & 58,8 \\
Canina & 1 & 2,9 & 2,9 & 61,8 \\
Premolar & 8 & 23,5 & 23,5 & 85,3 \\
Molar & 5 & 14,7 & 14,7 & 14,7 \\
TOTAL & 34 & 100,0 & 100,0 & 100,0
\end{tabular}

Tabla $N^{\circ}$ 5.- Ubicación de dientes Supernumerarios en Mandíbula

\begin{tabular}{cc}
\hline MAXILAR & MANDIBULAR \\
\hline 19 & 15
\end{tabular}

Tabla $N^{\circ}$ 6.- Prevalencia de dientes Supernumerarios en los Maxilares

En la presente investigación no se tomó en cuenta datos como la morfología de los dientes supernumerarios, debido a que las formas de los dientes son difíciles de visualizar radiográficamente, este dato solamente lo podríamos obtener posterior a la enucleación de los 
mismos; otro dato que no lo obtuvimos fue si se encontraron incluidos o erupcionados, ni tampoco se llevó un control de la extracción de los mismos por lo que se sugiere dar un correcto seguimiento a los pacientes que acuden con esta patología.

\section{Discusión.}

Vásquez D, 2012, (5) determinó mediante la obtención de datos en su estudio, una mayor prevalencia de dientes supernumerarios en el maxilar con 14 anomalías mientras que en la mandíbula solo 5; hecho que coincide con De Sousa-Gomes; et al, 2002, (3) quien en su estudio reportó 63 dientes supernumerarios en el maxilar y 53 en la mandíbula; Pinkham JR, 2001, (6) habla de mayor frecuencia para el maxilar con un 90 a 98\%; datos muy similares a nuestra investigación, la cual presentó en el maxilar superior el 57,14\% correspondiente a 20 dientes supernumerarios prevaleciendo con respecto a la mandíbula con el $42,85 \%$ equivalente a 15 dientes supernumerarios.

Raspall G, 2006, (7) afirma que el 90\% de pacientes presentan esta patología en la región anterior maxilar, dato que coincide con Alcántara C, 2005, (8) el mismo que menciona que la región anterior del maxilar superior es la más afectada por mesiodens; Regezi J, 1995, (9); Krüger E, 1987, (10) y Escoda G, 2010, (11) también comparten esta teoría con la presente investigación, la cual presentó el 47,1\% de dientes supernumerarios en la región premaxilar.En la mandíbula, Raspall G, (7) de igual manera encuentra el premolar inferior más común; Alcántara-Mena, (8) en su estudio también coloca a los premolares como el de mayor ocurrencia; datos que coincide con este trabajo investigativo, el cual observó que la región premolar en maxilar inferior tubo una prevalencia del $23,5 \%$. 
Vásquez D, (5) concluyó que la edad no es un factor determinante para la presencia de dientes supernumerarios, así lo demostró en su investigación, la cual presentó la primera y segunda década de vida la de mayor proporción con 7 dientes supernumerarios cada una. Datos que marcan una ligera diferencia con la presente investigación, ya que de acuerdo con los porcentajes descriptivos de nuestro estudio marca la segunda década de vida con 41,2\% como la de mayor prevalencia seguida por la tercera década de vida con el $35,3 \%$.

De Sousa-Gomes, (3) enunció en su artículo, una mayor proporción para el género masculino con 52 casos de hiperodoncia frente a 37 pertenecientes al género femenino, al igual que Chiapasco, Pinkham, Sánchez, Alcántara y Vásquez, $(1,6,12,8,5)$ en cuyo artículo investigativo muestra 13 DS en hombres y 6 en mujeres, mostrando que existe relación entre la patología y el sexo; teoría que concuerda con esta 38 investigación, la cual mostró, 24 (70,6\%) DS en hombres y 10 en mujeres equivalente a $29,4 \%$.

\section{Conclusiones.}

Determinamos que la prevalencia de dientes supernumerarios en el quirófano de la Facultad de Odontología de la Universidad Central del Ecuador, fue del 4\%.

Se estableció que los dientes supernumerarios se ubicaron con mayor frecuencia en maxilar que en mandíbula.

Se registró como la zona de mayor asiduidad en el maxilar superior, la zona incisiva, con los llamados mesiodens y en la mandíbula, predominó los dientes supernumerarios en la zona premolar. 
Se concluye que la edad no es un factor determinante para la presencia de dientes supernumerarios, debido a que no se tomó en cuenta su formación sino solamente su presencia, pudiendo evidenciarse en todas las décadas de vidas que se planteó en este estudio, presentándose con mayor frecuencia en la segunda década de vida.

Existió diferencia en la ocurrencia de hiperodoncia entre los sexos, siendo mayor en el sexo masculino que en el femenino.

\section{Bibliografía.}

1. Chiapasco M, Accardi A, Boisco M, Casentini P. Tácticas y Técnicas en Cirugía Oral. 2nd ed. Madrid: Amolca; 2010.

2. Alves N, Nascimento D, Olave E. Aspectos Clínicos y Morfológicos de los Dientes Supernumerarios. Int. J. Morphol. 2011 sep; 29(3): p. 1040-1046.

3. De Sousa-Gomes H, Gomes-Igor L. Frecuencia y distribución de dientes supernumerarios. Med Oral. 2002; 4(3): p. 84-87.

4. Hachity O, Bonilla R, Vázquez L, Peral G, Arenas M. Dientes supernumerarios múltiples. Presentación de caso clínico. Oral. 2012; 13(43): p. 927-930.

5. Vázquez D. Dientes supernumerarios: Estudio de prevalencia en la ciudad de Buenos Aires. Rev ADM. 2012; 69(5): p. 222-225.

6. Pinkham J. Odontología pediátrica. 3rd ed. Mac Graw-Hill Interamericana: Barselona; 2001.

7. Raspall G. Cirugía oral e implantología. 2nd ed. Madrid: Médica Panamericana; 2006.

8. Alcántara-Mena C. Prevalencia y distribucion de agenesias dentarias y dientes supernumerarios en pacientes de 7 a 18 años de edad atendidos en el Centro Médico Naval. Enero 2003 - Julio 2004. Tesis para obtar el Título Porfesional de Cirujano Dentista. Lima: Universidad Nacional Mayor de San Marcos, Facultad de Odontología; 2005.

9. Regezi J. Patología Bucal Mexico: Interamericana; 1995.

10. Kruger E. Técnicas Quirúrgicas para Odontologps Rio de Janeiro: Quintessence; 1987.

11. Escoda G. Cirugía Bucal Barselona: Oceano; 2010.

12. Sanchez J. Frecuencia de los dientes supernumerarios en poblacion infantil atendida en las clinicas de la facultad de odontología Mexicalide la UABC. Oral. 2009; 12(1). 